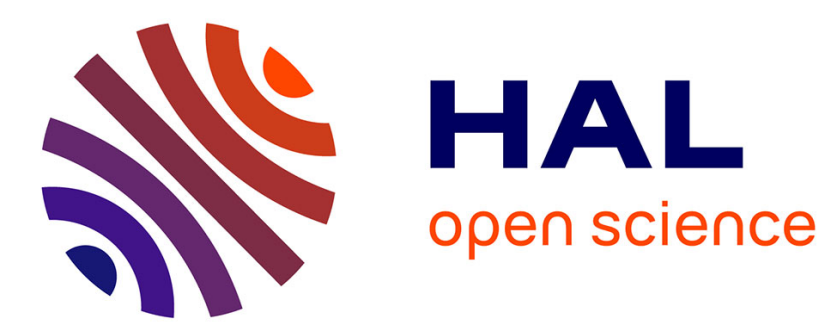

\title{
Photon bunching of the nonlinear photoluminescence emitted by plasmonics metals
}

\author{
Konstantin Malchow, Alexandre Bouhelier
}

\section{To cite this version:}

Konstantin Malchow, Alexandre Bouhelier. Photon bunching of the nonlinear photoluminescence emitted by plasmonics metals. Journal of the Optical Society of America B, 2021, 38, 10.1364/josab.411507 . hal-03388195

\section{HAL Id: hal-03388195 \\ https://hal.science/hal-03388195}

Submitted on 20 Oct 2021

HAL is a multi-disciplinary open access archive for the deposit and dissemination of scientific research documents, whether they are published or not. The documents may come from teaching and research institutions in France or abroad, or from public or private research centers.
L'archive ouverte pluridisciplinaire HAL, est destinée au dépôt et à la diffusion de documents scientifiques de niveau recherche, publiés ou non, émanant des établissements d'enseignement et de recherche français ou étrangers, des laboratoires publics ou privés. 


\title{
Photon bunching of the nonlinear photoluminescence emitted by plasmonics metals
}

\author{
Konstantin Malchow and Alexandre Bouhelier* (D) \\ Laboratoire Interdisciplinaire Carnot de Bourgogne CNRS UMR 6303, Université de Bourgogne Franche-Comté, 21000 Dijon, France \\ *Corresponding author: alexandre.bouhelier@u-bourgogne.fr
}

Received 1 October 2020; revised 16 December 2020; accepted 22 December 2020; posted 22 December 2020 (Doc. ID 411507); published 29 January 2021

\begin{abstract}
In this report, we investigate the statistical temporal distribution of nonlinear upconverted photoluminescence emitted by gold and silver nanostructures excited by focused near-infrared laser pulses. We systematically observe a clear signature of photon bunching regardless of the nano-object's geometry, material's crystalline arrangement, and electronic band structure. The similarity of the data obtained across very different plasmonic objects confirms that these types of nonlinear radiation share a common chaotic origin and result from a collection of emitters. The correlation of photons at a picosecond time scale released by nanoscale nonlinear sources of broadband radiation may be used in plasmonic devices to develop new data temporal and spatial processing functionalities. () 2021 Optical Society of America
\end{abstract}

https://doi.org/10.1364/JOSAB.411507

\section{INTRODUCTION}

When focusing a laser beam onto a noble metal, a broadband spectrum of emitted light is generally observed if the incident photon energy is sufficient to trigger interband excitations near the Fermi energy. This luminescence was first reported in 1969 by Mooradian [1] and is interpreted as the direct recombination of conduction-band electrons with $d$-band holes scattered in the electron wavevector space. Mooradian's linear luminescence was complemented over a decade later when reports of multiphoton luminescence emitted from gold surfaces were published [2,3]. The nearly quadratic dependence of the luminescence intensity to the incident pulsed laser power and the presence of a highenergy cutoff in the emission spectra suggested the involvement of two photons akin to a two-photon absorption process [3]. This upconverted nonlinear photoluminescence (NPL) features interesting spectral, spatial, and temporal properties. Its yield is particularly sensitive to local field enhancement [3]. The presence of localized surface plasmons favors the absorption of the laser pulse and create additional radiative decay channels [4,5]; the NPL response is thus very strong for resonant nanoscale objects [6-8]. Consequently, controlling local fields provides an elegant means to adjust the NPL emission rate $[9,10]$ and to shape the emitted spectrum $[11,12]$. Furthermore, mapping the NPL spatial distribution is a probe of the local optical density of states $[13,14]$ and a convenient way to visualize the surface plasmon modal landscape $[15,16]$. Finally, the temporal response of the broadband photoluminescence continuum presents an ultrafast few picoseconds lifetime that may be conveniently employed for fast signal modulation. An analysis of the decay dynamics highlighted differences from a coherent two-photon absorption process. The general consensus to understand the absorption process is a step-like sequential intake of two photons via the mediation of a metastable state [17-20]. All of these peculiar attributes found interesting application venues. For instance, NPL generated by miniature plasmonic structures offers a technological route to produce integrated nonlinear functionalities such as local wavelength conversion devices with ultrafast kinetics [21] or computing Boolean operations [22]. NPL can also be used for label-free biological imaging [23].

In recent years, the two-photon induced photoluminescence interpretation has been challenged by experimental observation of high-order dependencies involving integer and noninteger exponents $[8,18,24]$, which in some cases are measured up to the seventh order [21]. In a 2015 article, Haug and co-workers proposed a different interpretation where the nonlinearity of the process not only arises from the absorption process, but also from the peculiarity of the emission [25]. In this school of thought, the luminescence emerges from intraband recombinations of conduction electrons brought at elevated temperatures, a mechanism especially dominant for high pump irradiance [26,27]. Such radiative decay of a nonequilibrium electron distribution also has been observed in electrically pumped devices [28-31]. The interest here lies in the possibility to use NPL as a nanoscale thermal source of broadband photons with picosecond kinetics.

Motivated by this body of observations, we confirm in this work the intrinsic nature of the NPL source by measuring the statistical temporal distribution of the photons. We decide to focus our measurement on upconverted nonlinear photons. We expect to observe bunching also for the luminescence emitted 
in the downconverted spectral window, because it would also stem from a chaotic process and therefore be bunched, irrespective of the underlying emission mechanisms. We quantify the second-order correlation function $g^{(2)}(\tau)$, which describes the correlation between two optical signals separated by a time difference $\tau=t^{\prime}-t^{\prime \prime}$. We investigate a set of generic plasmonic objects with different material compositions $(\mathrm{Ag}, \mathrm{Au})$, atomic organizations (crystalline, amorphous), and geometries [film, nanoparticles, two-dimensional (2D) flakes] to unequivocally validate the chaotic nature of the emission. In all of our experiments, we observe a clear photon bunching peak of the nonlinear photoluminescence characterized by a $g^{(2)}(\tau=0)>1$, which confirms the expected response stemming from an ensemble of emitters.

\section{EXPERIMENTAL PROCEDURES}

Our experimental approach to measure the photon correlation of the nonlinear photoluminescence is shown in Fig. 1. We use a pulsed Ti:sapphire laser (Chameleon, Coherent, Inc.) delivering $180 \mathrm{fs}$ pulse duration at a repetition rate of $80 \mathrm{MHz}$ and tuned to a wavelength $\lambda=808 \mathrm{~nm}$. The output power of the laser is controlled by a polarization beam splitter and two half-wave plates (Thorlabs, Inc). We spectrally filter the beam with a $808 \mathrm{~nm}$ line filter (Semrock, Inc.) to remove residual laser luminescence emitted at shorter wavelengths. The beam is then sent into an inverted microscope (Eclipse, Nikon) equipped either with a dichroic beam splitter (Chroma, Inc.) to conduct the photon statistics or with a 50/50 beam splitter to measure the power dependence of the different spectral regions. We adjust the laser beam diameter with a beam expander to overfill the entrance pupil of an oil immersion objective lens $(100 \times$, 1.49 numerical aperture). The beam is thus focused to the diffraction limit on the objects under scrutiny. The samples are all prepared on standard glass cover slips and can be raster scanned in three directions by a piezoelectric stage (Mad City Labs, Inc). The generated NPL induced by the laser beam is collected with the same objective and separated from the incident laser light by the dichroic beam splitter or by a set of two notch filters (Semrock, Inc.). The NPL typically spans the entire visible region of the electromagnetic spectrum. Representative spectra are illustrated later on in the discussion. The NPL signal is then directed to a Hanbury Brown and Twiss interferometer (HBT) to observe photon correlations. To increase the contrast between the residual background signal and the photon correlation histogram [32,33], we select a small spectral region of the NPL with a narrowband line filter (Melles Griot, Inc.) centered at $632.8 \mathrm{~nm}$ with a spectral width of $4 \mathrm{~nm}$ before sending the light to the HBT. In the interferometer, the NPL is separated in two by a $50 / 50$ beam splitter and each arm is focused on an avalanche photodiode (APD), with $50 \mu \mathrm{m}$ detection area, $30 \mathrm{ps}$ time resolution and $77 \mathrm{~ns}$ dead time (Micro Photon Devices). To eliminate the afterglow contribution generated by the APDs from the photon correlation histogram [34], we introduced a pinhole and a short pass filter $(714 \mathrm{~nm})$ before each detector. Both APDs are then electrically connected to a time-correlated single photon counting module (HydraHarp, PicoQuant $\mathrm{GmbH}$ ). The module registers a timestamp for each photon event detected per APD. When needed, the NPL collected by

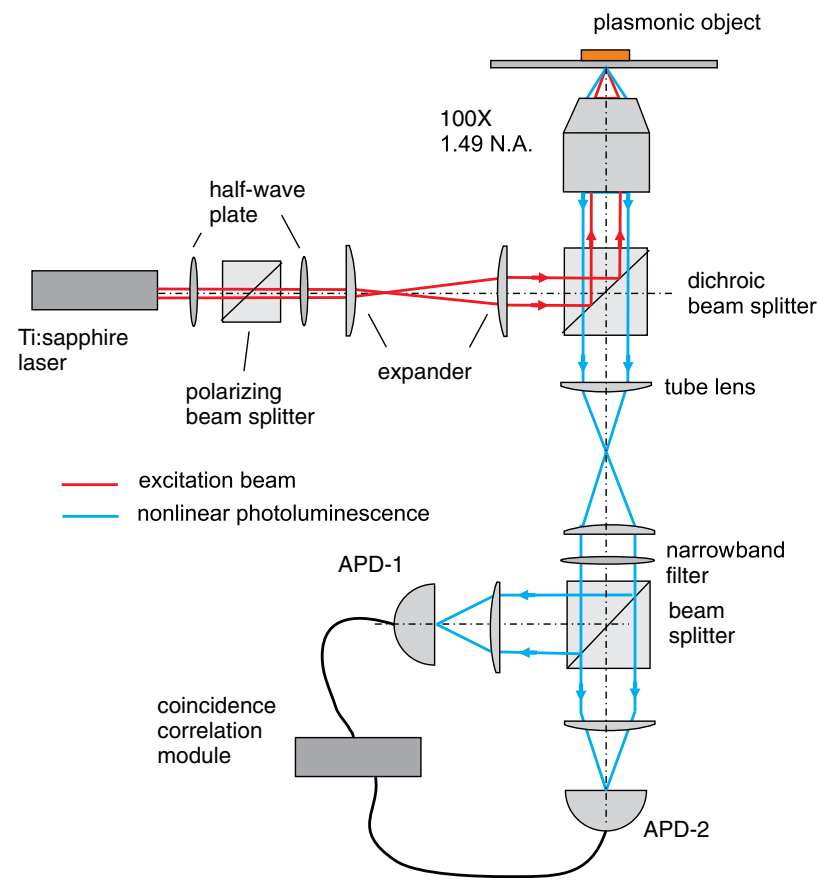

Fig. 1. Simplified sketch of the experimental setup used to excite the nonlinear photoluminescence (blue rays) from plasmonics objects excited with a focused femtosecond laser oscillator (red rays). The photon statistics is measured with a correlation module registering the coincidences from the two APDs.

the objective lens is directed to an alternate output port of the microscope (not shown) equipped with a grating spectrometer (Shamrock 300i, Andor).

\section{EMISSION MECHANISMS}

Figure 2 shows where to situate the response of the nonlinear activity with respect to incident power in view of the discussion in [26]. To this aim, we measure the spectral amplitude of the upconverted and downconverted photoluminescence emitted from a Au film as a function of the temporal averaged incident laser intensity. For each intensity level, 10 spectra are acquired and averaged. Figure 2(a) illustrates the amplitude of the detected light for two wavelengths in the upconverted region $(\lambda=600 \mathrm{~nm}$ and $\lambda=750 \mathrm{~nm})$ and one taken in the downconverted part $(\lambda=950 \mathrm{~nm})$. The data points in this double logarithmic plot highlight a power law dependence with an exponent $p$ given by the slope of the fit to the data (solid lines). Figure 2(b) gathers the values of the exponent $p$ across the detected spectral window encompassing the upconverted and downconverted regions. We excluded from the data analysis the spectral range 730-920 $\mathrm{nm}$ because of an incomplete laser rejection and Raman contributions from the substrate. The size of the error bars increases in the short wavelength region because of the reduced signal emitted for the Au film. The graph unambiguously reveals two different trends for the power exponent. For the downconverted region, the exponent stays constant at $p \sim 1$. This is consistent with an earlier report [9] as well as the measurement of Roloff et al. taken at reduced excitation levels [26]. The linear evolution of the exponent with a decreasing wavelength in the upconverted window is also in agreement 

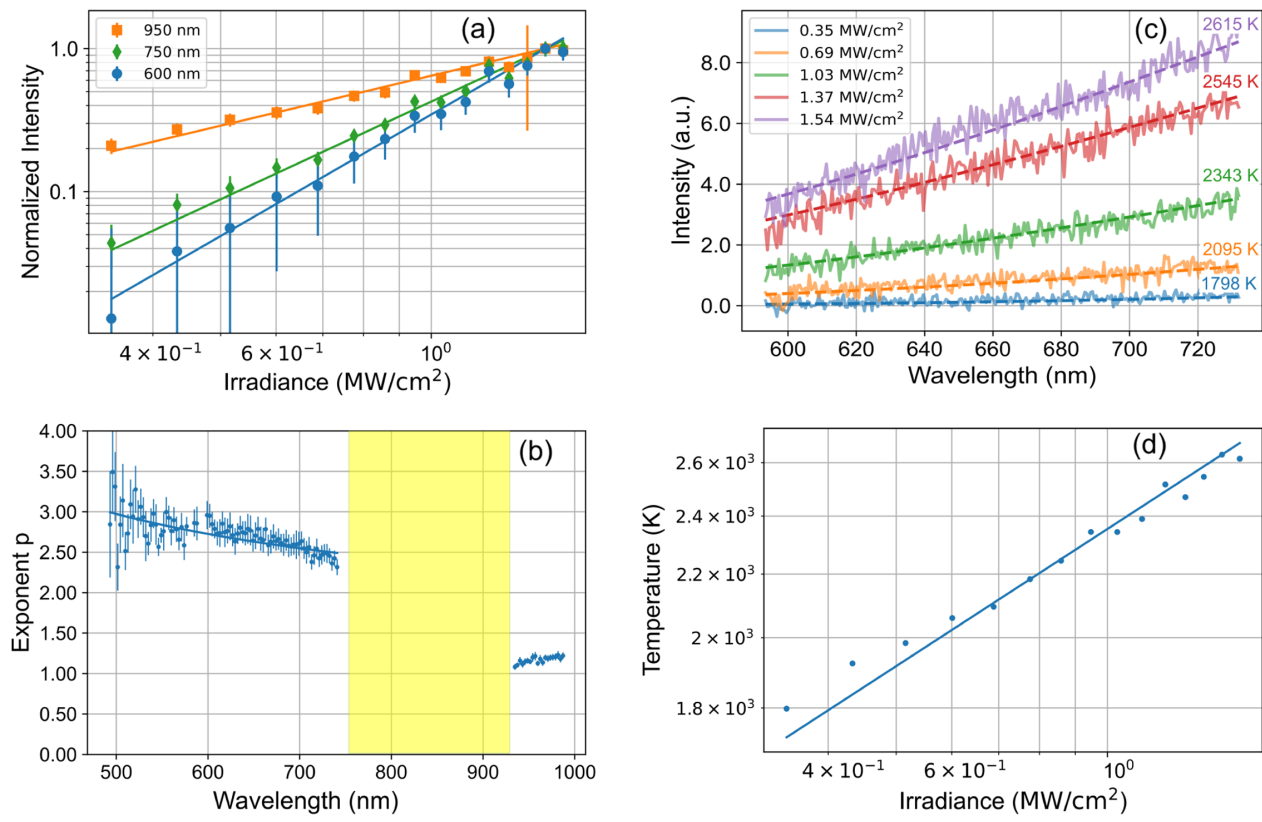

Fig. 2. (a) Dependence of the photoluminescence signal of a Au film with incident laser intensity taken at two up-converted wavelengths (600 nm and $750 \mathrm{~nm}$ ) and one down-converted wavelength $(950 \mathrm{~nm})$. The slope of the linear fits in the double logarithmic plot is used to extract the power exponent $p(\lambda)$ displayed in (b). $p$ scales linearly in the nonlinear emission regime and stays at $\sim 1$ for the downconverted side. The shaded area is removed from the analysis as its contains the remaining laser signal and some Raman contributions from the subtrate. (c) NPL spectra taken at different incident laser intensities. The dotted lines are fits obtained by applying Planck's law of radiation with electronic temperature $T_{\mathrm{e}}$ set as a fitting parameter. (d) Evolution of the deduced electronic temperature with laser irradiance.

with the literature. This discards NPL as a two-photon interband transition mechanism in our measurements ( $p$ would be constant at 2) and attests the contribution of an heated electronic distribution by the femtosecond laser pulses $[21,26]$. We tentatively deduce the temperature of the electrons by fitting the spectra [25] taken at different irradiance levels with Planck's law,

$$
I\left(\lambda, T_{\mathrm{e}}\right)=\phi \frac{2 \pi c}{\lambda^{4}} \frac{1}{e^{\frac{h c}{\lambda k_{\mathrm{B}} T_{\mathrm{e}}}}-1},
$$

where $I$ is the spectral photon flux, $h$ is Planck's constant, and $k_{\mathrm{B}}$ is Boltzmann's constant. $T_{\mathrm{e}}$ is the electron temperature and is a variable of the fit. $\phi$ is another free parameter taking into account the detection efficiency of the setup and the local density of optical states. The solid lines in Fig. 2(c) are fits using Eq. (1) to a selected a series of experimental spectra taken at various incident irradiance. The fits give a reasonable agreement with the electronic temperatures indicated next to the spectra and a constant $\phi$ coefficient for all fits. The electronic temperatures are consistent with reported values in the literature $[20,21,25,26,35]$. We plot in Fig. 2(d), the evolution of $T_{\mathrm{e}}$ with laser irradiance $I_{\text {laser }}$.

For bulk metal, and in the limit of $k_{\mathrm{B}} T_{\mathrm{e}} \ll \epsilon_{F}$, where $\epsilon_{F}$ is the Fermi energy, the maximum electron temperature at the end of pulse can be estimated [35] by equating the energy of the electron gas $W_{\mathrm{e}}=C_{\mathrm{e}} n_{\mathrm{e}} T_{\mathrm{e}}=\pi^{2} n_{\mathrm{e}} k_{\mathrm{B}}^{2} T_{\mathrm{e}}^{2} / 2 \epsilon_{F}$ with the energy density absorbed by the metal $W_{\mathrm{abs}}=2 A F_{\text {laser }} / \delta$. Here $\delta$ is the skin depth, $A$ is Fresnel's absorption coefficient, $F_{\text {laser }}$ is the time-integrated laser energy over the pulse duration, $C_{\mathrm{e}}$ is the heat capacity of the electrons, and $n_{\mathrm{e}}$ is the electron density. The electron temperature is thus a quadratic function of the laser irradiance with $T_{\mathrm{e}}^{2} \propto I_{\text {laser }}$. Figure 2(d) suggests a linear dependence in this $\log -\log$ representation indicating that $T_{\mathrm{e}}^{a} \propto I_{\text {laser }}$ with $a=3.4$. This value departs from the expected free-electron quadratic dependence, but is consistent to the mean values reported in [25], $(a=4)$. The deviation was explained by the different electronic relaxation rates occurring in nanoscale geometries.

\section{PLASMONIC OBJECTS}

We move on now to interrogate the photon statistics of the NPL released from various plasmonic objects to confirm the general attribute of the nonlinear signal regardless of the constituent material, the geometry, and the crystalline nature. Three different types of object are used for this experiment. The first type of object is patterned by UV lithography and a liftoff procedure to produce micron-scale metal patches. Each patch consists of a $50 \mathrm{~nm}$ thick Au film deposited by physical vapor deposition in a vacuum chamber. The adhesion of the metal layer on the glass substrate is promoted using a $2 \mathrm{~nm}$ thick Ti layer. The films are amorphous with a surface roughness typically measured at 2 to $5 \mathrm{~nm}$. The patches are large enough to be considered as films and do not feature any well-defined wavelength resonances. On the same substrate, we then deposit Au nanoparticles (BBI Solutions) with a mean diameter of $60 \mathrm{~nm}$. We dilute the asreceived nanoparticles in an aqueous solvent and drop-cast them on the glass cover slip patterned with the metal patches with a dispersion allowing single-particle measurement. We isolate and identify single nanoparticles from clusters by scanning electron microscopy with the help of landmark patches. We then finalize 
this gold-based sample by drop-casting atomically flat singlecrystal micrometer-large Au flakes [16,36]. These large objects possess a thickness-dependent electronic band structure [37]. For the sake of the completeness of the study, we also produced $50 \mathrm{~nm}$ thick patches of Ag using the same methodology.

Figure 3(a) is a confocal map of the NPL signal when a $\mathrm{Au}$ patch and single Au nanoparticles are raster scanned through the focal area. The map is constructed by measuring the NPL photon counts registered by one of the two APD for each $(x, y)$ position of the sample. For this type of experiments, the narrowband filter in front of the APD is removed so that the detector measures the total number of photons emitted in the entire detection spectral window. The linear polarization of the laser is aligned vertically and the average laser power at the focus is $4 \mathrm{MW} \cdot \mathrm{cm}^{-2}$. As already reported in many instances in the literature, the NPL emission is highest at the edges of the patch because the spatial discontinuity provides a local field enhancement and creates additional momentum. Figure 3(b) is the NPL spectrum obtained from the region indicated by the circle in Fig. 3(a). The spectrum has a monotonous decaying tail toward short wavelength, typically reported in the literature, and is consistently observed in other areas of the patch. The long wavelength cutoff results from the transmission edge of the dichroic beam splitter, as shown as a gray curve in the spectrogram. A second-harmonic response occurring at $404 \mathrm{~nm}$ is also ubiquitous in the collected spectra. In our experiments, this second-harmonic yield is generally much weaker than the NPL intensity. The spatial NPL distribution measured by the
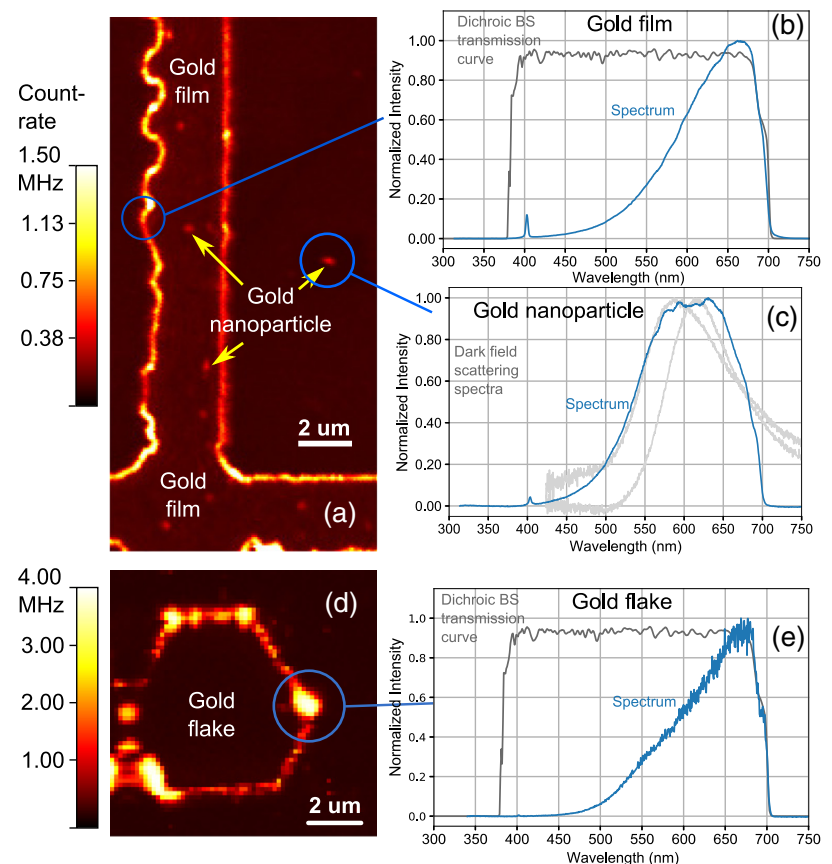

Fig. 3. (a) NPL confocal map obtained by raster scanning the $\mathrm{Au}$ patches and $60 \mathrm{~nm}$ nanoparticles in the focus. (b) and (c) Normalized NPL emitted spectra (blue curve) measured at the locations indicated by the arrows in (a). The gray curve in (b) is the transmission curve of the dichroic beam splitter (BS) and the gray curves in (c) are two representative dark-field spectra of Au nanoparticles used here. (d) NPL confocal map obtained on a gold flake. Its corresponding spectrum is shown in (e). All spectra are normalized by their peak value (blue curves) after a background subtraction. silver film and the corresponding spectrum are qualitatively very similar to the map and spectrum obtained from the Au film, so they are not shown.

In Fig. 3(a), individual nanoparticles sitting on the glass substrate and on the film are also emitting a similar NPL yield so they can be easily distinguished in the confocal map. There are a few isolated particles in the scan window; some of them are indicated by the arrows. Figure 3(c) displays the NPL spectrum of the single Au nanoparticle identified in Fig. 3(a). A broad resonance shapes the NPL response. The resonance is the tell-tale signature of the presence of a localized surface plasmon in the nanoparticle [11]. The two gray curves in Fig. 3(c) are linear scattering spectra of two isolated nanoparticles situated in an adjacent area. The spectra are acquired with dark-field illumination. Although varying from nanoparticle to nanoparticle, the scattering surface plasmon peaks broadly match the NPL maximum. The last set of data pictured in Figs. 3(d) and 3(e) are the confocal NPL map and emitted NPL spectrum recorded on a single-crystal flake. The NPL is mostly emitted at the edges and apices of the quasi-2D structure [16]. Similar to the gold stripe, the origin of this strong response located at the boundaries is the combining results of the enhancement of the electromagnetic field at the dielectric discontinuity and the particularity of the electronic band structure around the specific atomic orientation of the edges and apices [38].

\section{SECOND-ORDER CORRELATION}

Next, we investigate the temporal statistical distribution of the NPL by measuring the second-order correlation function. The second-order temporal correlation of a photon source is defined as [39]

$$
g^{(2)}(\tau)=\frac{\langle\mathrm{I}(t) \mathrm{I}(t+\tau)\rangle}{\langle\mathrm{I}(t)\rangle\langle\mathrm{I}(t+\tau)\rangle},
$$

where $I$ is the intensity of the source and the brackets indicate averaging over fluctuating quantities. The photon correlation function is related to the probability to detect a photon at time $t+\tau$ given a photon already detected at time $t$ [40]. For sufficiently large time delays $\tau \rightarrow \infty$ any correlation between photon time arrivals is lost and $g^{(2)}=1$. At shorter time delays, the photon correlation depends on the properties of the source. For single-photon sources, $g^{(2)}(0)<0.5$ since it is not possible to absorb a photon twice. On the contrary, for chaotic sources such as thermal emitters, a bunching peak with $g^{(2)}(0)>1$ indicates that photons preferably arrive together. For coherent sources instead, the intensity fluctuations are independent and therefore $g^{(2)}=1$ at all time delays.

With the time stamps recorded by both APDs and by using the time-correlated single counting module we calculate the start-stop coincidence counts and build a histogram of the time delays between the first APD registering a photon and the second APD registering the next photon. This procedure is similar to a time-to-amplitude conversion. For time delays shorter than the dead time of the APDs, this is equivalent to the full correlation but is less calculation intensive $[40,41]$.

The histogram measured for the Au film is shown in Fig. 4(a). The NPL is collected from the region indicated by the circle in Fig. 3(a) to maximize the detected NPL counts and number of 

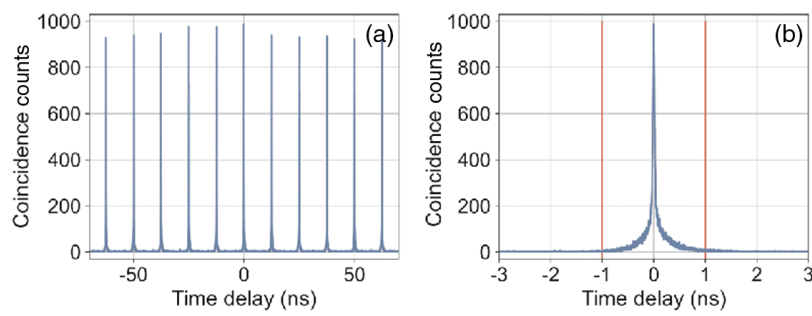

Fig. 4. (a) Histogram of coincidence counts of the nonlinear luminescence emitted by a gold film. The measurement recorded the correlation for $1 \mathrm{~h}$. (b) Time frame around the first peak at zero delay. The red lines show the integration limits.

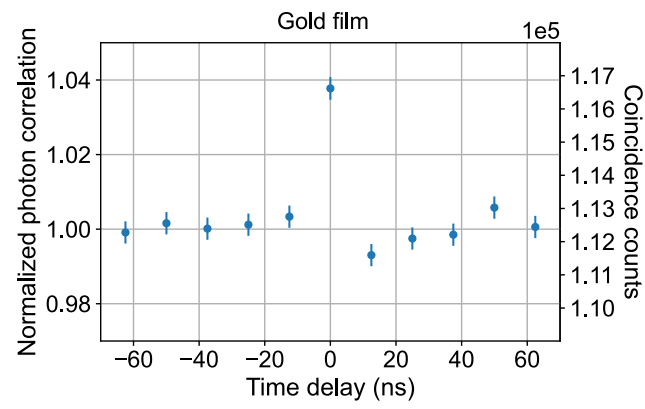

Fig. 5. Normalized second-order correlation function $g^{(2)}$ of the nonlinear photoluminescence emitted by a Au film. The right axis shows the total coincidence counts extracted from the analysis of the peaks in Fig. 4(a).

coincidences. The histogram is constructed from a $1 \mathrm{~h}$ acquisition time. Because we are using a pulsed laser, the coincidence histogram shows discrete peaks spaced uniformly, with the separation time given by the inverse of the laser's repetition rate. In our analysis, we sum up the total counts of each peak integrated within a temporal window of $2 \mathrm{~ns}$ centered at the peak. This is exemplified in Fig. 4(b) for the central peak at zero delay. The integration window is outlined by the red vertical lines.

To calculate the $g^{(2)}(\tau)$, we normalized the coincidence count histogram by the mean of the intensity correlations estimated within the dead time of the detectors and exclude data near the zero time delay. Figure 5 displays the $g^{(2)}$ function of the NPL emitted from the Au film. Each data point corresponds to the total coincidence counts of a peak, as shown in Fig. 4. The value of the photon correlation at zero time delay extracted from the graph is $g^{(2)}(\tau=0)=1.038 \pm 0.004$, which unambiguously confirms a photon bunching of the nonlinear photoluminescence. The error bars in the graphs are indicative of the standard deviation.

The expected bunching value (see Appendix A) for a pulsed chaotic source is given by

$$
g^{(2)}(0)=1+C \cdot \sqrt{\pi} \cdot \frac{\tau_{\mathrm{coh}}}{2 \cdot t_{\mathrm{p}}},
$$

where $\tau_{\mathrm{coh}}$ and $t_{\mathrm{p}}$ are, respectively, the coherence time and the decay time of the light source, and $0 \leq C \leq 1$ is the so-called coherence factor [32], which takes into account the reduced spatial coherence, the polarization of the light, and can also account for background noise. We determine $\tau_{\text {coh }}=0.122$ ps by Fourier transforming the transmission spectrum of our narrowband line filter placed before the HBT. The NPL lifetime has been reported to be about 1 ps $[9,18,20]$; thus, we expect to observe $g^{(2)}(0)-1=C \cdot 0.10$. This implies $C=0.34$ in our experiment. This value is not too far from $C=0.5$, which is the maximum value of the coherence factor for measurements conducted with unpolarized light. Demichel et al. showed that the relaxation of the hot carriers depend on the incident energy, or equivalently to the temperature of the electronic distribution. Values for the lifetime of the hot electrons were reported to vary between 0.6 ps to 2 ps, depending on the laser irradiance [20]. A modified $t_{\mathrm{p}}$ changes the estimation of coherence factor $C$ in Fig. 5 to a value $C=0.17$ if we consider a fast relaxation $t_{\mathrm{p}}=0.6$ ps to $C=0.68$ for $t_{\mathrm{p}}=2 \mathrm{ps}$. Since we are not observing a polarized NPL signal from the Au film, $C$ is bound at 0.5 , which in turns puts a limit at the emission decay in our measurement at $t_{\mathrm{p}}=1.4 \mathrm{ps}$.

The value of $g^{(2)}(0)$ can be augmented by polarizing the detected light [32], increasing the spatial coherence and the time resolution $\tau_{\text {det }}$ of the detection $[32,42,43]$, and reducing the environmental background count contribution [40]. The integration time $T_{o}$ to distinguish the bunching peak from the background with a given signal-to-noise ratio (SNR) is for a pulsed source given by

$$
T_{o}=\frac{\mathrm{SNR}^{2}}{C^{2}} \cdot \frac{4 \cdot \tau_{\mathrm{p}}^{2} \cdot f_{\text {rep }}}{I_{1}^{\text {mean }} \cdot I_{2}^{\text {mean }} \cdot \pi \cdot \tau_{\mathrm{coh}}^{2}},
$$

where $I_{1}^{\text {mean }}, I_{2}^{\text {mean }}$ is the mean light intensity at detector 1 resp. 2, and $f_{\text {rep }}$ is the repetition rate of the pulsed source. Equation (4) is valid if $\tau_{\mathrm{coh}} \ll t_{\mathrm{p}}$. By spectral filtering, one can increase the bunching peak height without increasing the integration time as long as the product $I^{\text {mean }} \cdot \tau_{\text {coh }} \propto \frac{I^{\text {mean }}}{B}$ is constant, where $B$ is the bandwidth of the spectrum. However, if the photon rate per unit bandwidth decreases by additional filtering, the integration time increases quadratically. In our experiment, using a bandpass filter centered at $632.8 \mathrm{~nm}$ with a bandwidth of $4 \mathrm{~nm}$ provides an acceptable experimental tradeoff. Finally, the time resolution of the detectors are not playing any role in the height of the bunching peak as long as it remains significantly smaller than the repetition period of the pulse train.

In the following section, we investigate the robustness of the photon statistics by analyzing the coincidence counts of the NPL emitted by other types of plasmonic object introduced in Figs. 3(a)-3(c). In all measurements, we observe a clear bunching peak, as demonstrated in Fig. 6. For the Au nanoparticle and the silver film, we observe a bunching value of, respectively, $g^{(2)}(0)=1.045 \pm 0.016$ and $g^{(2)}(0)=1.037 \pm 0.007$, two values similar to the gold film. The presence of a broad surface plasmon resonance in the nanoparticle or the nature of the metal is therefore not affecting the NPL bunching peak. Interestingly, the bunching peak for the Au flake reaches $g^{(2)}(0)=1.070 \pm 0.004$, higher than $g^{(2)}(0)=1.05$, which is the maximum bunching value we expect for our measurements with unpolarized light. As discussed above, higher $g^{(2)}(0)$ peak may be due to a partial polarization of the emitted NPL or a shorter lifetime of the response. Conducting a polarization analysis of the NPL response reveals that the NPL is essentially unpolarized. We therefore hypothesize that the lifetime of NPL emitted by crystalline flakes may be shorter than 1 ps. This is not 

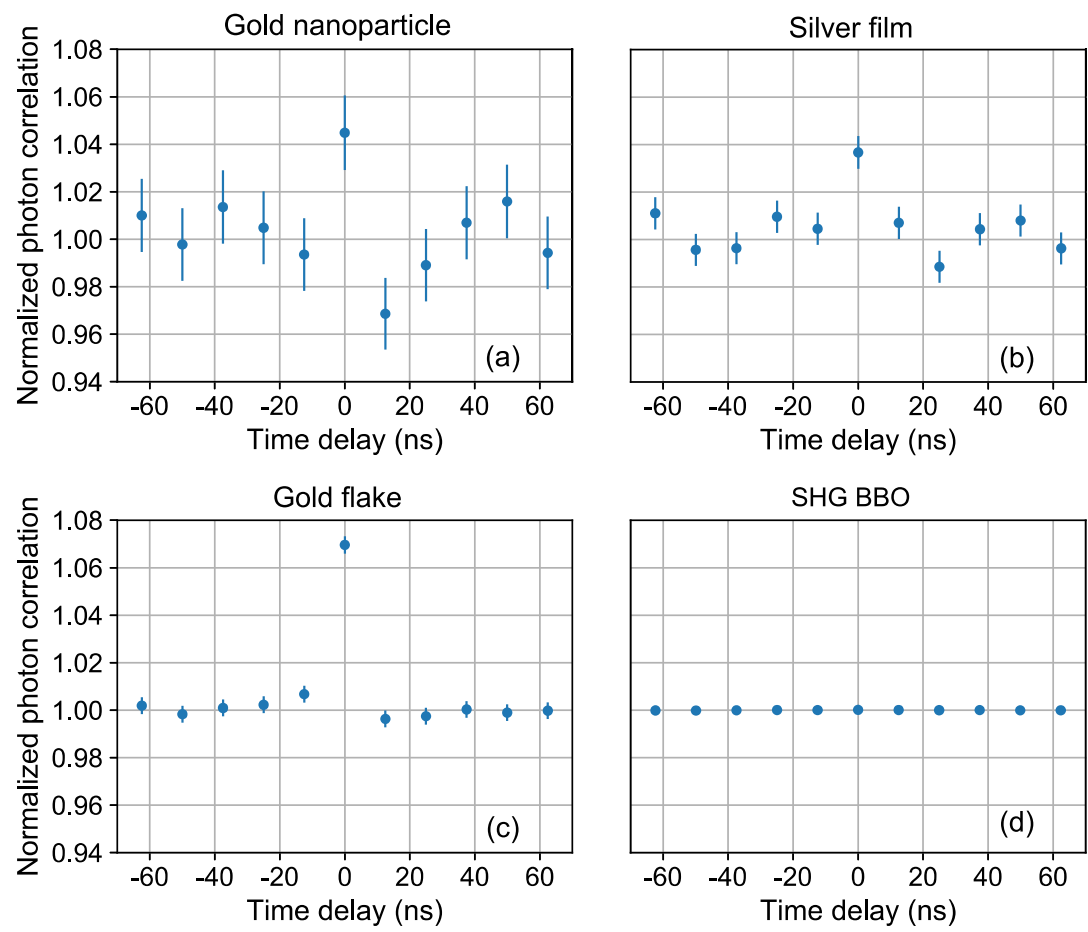

Fig. 6. Normalized second-order correlation function $g^{(2)}$ of the nonlinear photoluminescence emitted by (a) 60 nm gold nanoparticle; (b) rough Ag film; and (c) single-crystal flake. (d) Sanity check experiment displaying $g^{(2)}$ measured by recording the second-harmonic generated by a phasematched nonlinear $\beta$-barium borate (BBO) crystal.

surprising because the NPL spectrum released by the Au flake does not show any marked resonance [Fig. 3(e)] and the lifetime of the nonlinear response has been shown to significantly increase for resonant objects [20]. We note that the NPL signals emitted by these different metal structures are plagued by signal variations during the hour-long measurement at high irradiance. Slow variations of the NPL intensity (scale of seconds to minutes) are consequent to inherent lateral drifts of the probed area outside the focal region. These changes are user corrected in operando to maintain the NPL count rate maximum. We also observed fast changes of the NPL, especially when probing nanoparticles. These rapid fluctuations are associated with the inevitable morphological change of the structure subject to such an intense laser pulse irradiance [11]. Upon completion of the measurement, optical and scanning electron microscopy inspections of the nanoparticles under scrutiny confirmed the laser-induced damages. However, reshaping of a nanoparticle or a Au film is typically associated with a decreasing NPL signal amplitude $[11,44]$, so that most of the coincidence counts measured to estimate the $g^{(2)}(\tau)$ were collected before the structures suffered from severe morphological modifications. Since we consistently measure a similar bunching statistic across all the tested objects, we believe that a modification of the morphology during the measurement has no effect on the chaotic nature of the emission.

Finally, we verified our measurement protocol by analyzing the photon correlation of the second harmonic of the laser wavelength. Second harmonic generation (SHG) is produced by passing the Ti:Saph laser beam inside a nonlinear dielectric crystal. The phase-matching condition is obtained by adjusting the cut axis of the crystal with respect to the incident beam. As expected, only a very weak photon bunching $g^{(2)}(0)=1.00014 \pm 3 \times 10^{-5}$ is measured for this parametric coherent process $[39,45]$ and is not visible in Fig. 6(d) if we keep the same vertical axis in all graphs.

\section{CONCLUSIONS}

We observe a clear confirmation of photon bunching of the nonlinear photoluminescence emitted from different gold and silver nanostructures. The observed bunching peak in the secondorder correlation function are between $1.038<g^{(2)}<1.07$. The presence of systematic $g^{(2)}(0)>1$ regardless of the nature of the object (material, geometry, or crystallinity) validates that the emitted signals share the same intrinsic origin. There are different possible strategies to engineer the photon correlation; for instance, by increasing the coherence time of the NPL through a shaping of the local density of states [33,46]. An interesting approach in this context would consist of integrating the gold structures within a high-quality dielectric resonator. This would condition photon emission into spectrally narrow resonances while the total emitted intensity should stay constant [47]. Provided we can neglect re-absorption, this would lead to both an increase of the bunching peak and a decrease of the integration time. The advantage of deploying NPL as source of bunched light resides in its integration with a photonic platform. This allows the transposition of some far-field applications of bunched light into the nanometer scale to then explore new territories such as temporal ghost bunching [48] and spatial correlations mediated by plasmonic landscapes. 


\section{APPENDIX A: THEORETICAL BUNCHING PEAK HEIGHT AND INTEGRATION TIME FOR PULSED SOURCES}

The number of total coincident counts $C_{\text {tot }}$ is given by [39]

$$
\begin{aligned}
C_{\mathrm{tot}}\left(T_{\mathrm{o}}\right)= & \int_{T_{\mathrm{o}}-\frac{\Delta \tau}{2}}^{T_{\mathrm{o}}+\frac{\Delta \tau}{2}} \mathrm{~d} \tau \int_{0}^{T_{\mathrm{o}}} \mathrm{d} t \int_{-\infty}^{\infty} \mathrm{d} t^{\prime} \int_{-\infty}^{\infty} \mathrm{d} t^{\prime \prime} \\
& \cdot I_{1}\left(t^{\prime}\right) I_{2}\left(t^{\prime \prime}\right) \cdot D_{1}\left(t^{\prime}-t\right) D_{2}\left(t^{\prime \prime}+\tau-t\right) \\
& \cdot\left(1+C \cdot\left|g^{(1)}\left(t^{\prime}-t^{\prime \prime}\right)\right|^{2}\right) \\
= & C_{r}+C_{b}\left(T_{\mathrm{o}}\right) .
\end{aligned}
$$

$\Delta \tau$ is the time bin of the coincidence histogram, $T_{\mathrm{o}}$ is the integration time, $D_{1}(t)$ and $D_{2}(t)$ are the detectors instrument time response functions, $I_{1}$ and $I_{2}$ are the intensities detected by the detectors, and $g^{(1)}$ is the normalized first-order coherence. The total coincidence counts can be separated into random coincident counts $C_{r}$, as expected from a laser source, and $C_{b}$, which are the additional bunching counts.

Equation (A1) can be integrated both for a constant and pulsed sources, as already shown in $[32,49]$. For a pulsed source, we have

$$
C_{r}=I_{1}^{\text {mean }} \cdot I_{2}^{\text {mean }} \cdot \frac{T_{\mathrm{o}}}{f_{\text {rep }}},
$$

where $I_{1,2}^{\text {mean }}$ are the mean intensities at detector 1 and 2 . We set the time bin $\Delta \tau$ large enough to integrate over the pulse width in the histogram, as illustrated in Fig. 4. In the following, we consider that $\left|g_{1}(\tau)\right|^{2}=\exp \left(-\frac{\tau^{2}}{\sqrt{2} \tau_{\text {coh }}}\right)$. We further assume exponentially decaying pulses with lifetime $t_{\mathrm{p}}$, as expected for a thermal source and for the NPL in gold [20]. We thus model our pulsed NPL source as

$$
I_{1,2}(t)=\sum_{n} I_{1,2}^{\text {mean }} \cdot I_{\mathrm{p}}\left(t-n \cdot \frac{1}{f_{\text {rep }}}\right),
$$

with

$$
I_{\mathrm{p}}(t)=\left\{\begin{array}{ll}
0 & \text { for } t<0 \\
\frac{1}{t_{\mathrm{p}}} e^{-\frac{t}{t_{\mathrm{p}}}} & \text { for } t \geq 0
\end{array},\right.
$$

and insert this into Eq. (A1). For the instrument response functions $D_{1,2}(t)$, we assume that $\int_{-\infty}^{\infty} D_{1,2}(t) \mathrm{d} t=1$ and that $D_{1,2}(t)=0$ for $|t|>\frac{0.1}{f_{\text {rep }}} \gg t_{\text {det }}$, where $t_{\text {det }}$ is the detector's time resolution. We obtain

$$
\frac{C_{b}}{C_{r}}=C \cdot \sqrt{\pi} \cdot a \cdot(1-\operatorname{erf}(a)) \cdot e^{a^{2}} \simeq C \cdot \sqrt{\pi} \cdot \frac{\tau_{\mathrm{coh}}}{2 \cdot t_{p}},
$$

with $a=\frac{\tau_{\text {coh }}}{2 \cdot t_{p}} \quad$ and $\quad \operatorname{erf}(x)=\frac{2}{\sqrt{\pi}} \int_{0}^{x} e^{-t^{2}} \mathrm{~d} t$. Inserting $\tau_{\text {coh }}=0.122$ ps and $t_{p}=1 \mathrm{ps}$, gives $C \cdot 0.10$.

As shown in [49], the signal-to-noise ratio (SNR) of the extra bunching counts to the random background counts is given by

$$
\mathrm{SNR}=\frac{C_{b}}{\sqrt{C_{r}}}=\frac{C_{b}}{C_{r}} \sqrt{C_{r}},
$$

from which we can obtain the integration time,

$$
T_{\mathrm{o}}=\frac{\mathrm{SNR}^{2}}{\left(\frac{C_{\mathrm{b}}}{C_{\mathrm{r}}}\right)^{2}} \cdot \frac{f_{\mathrm{rep}}}{I_{1}^{\text {mean }} \cdot I_{2}^{\text {mean }}}
$$

Inserting $f_{\text {rep }}=80 \mathrm{MHz}, I_{1,2}^{\text {mean }}=10^{4} \mathrm{~Hz}, \frac{C_{\mathrm{b}}}{C_{\mathrm{r}}}=0.034$, and $\mathrm{SNR}=3$, we obtain an integration time $T_{\mathrm{o}}=1.73 \mathrm{~h}$, in agreement with what we observe experimentally.

Funding. Institut de physique; European Regional Development Fund (program Optiflex); Conseil régional de Bourgogne-Franche-Comté (program APEX); Délégation Régionale à la Recherche et à la Technologie; Agence Nationale de la Recherche (EIPHI Graduate School ANR-17-EURE-0002).

Acknowledgment. Device fabrication was performed in the technological platform ARCEN Carnot. The authors thank Florian Dell'Ova and Dr. Erik Dujardin (CEMES, Toulouse) for preparation of the gold flakes, Dr. Adrian Agreda for his help producing gold films, and J. Jakob S. Kunjappy for his help during the photon correlation measurements.

Disclosures. The authors declare no conflicts of interest.

\section{REFERENCES}

1. A. Mooradian, "Photoluminescence of metals," Phys. Rev. Lett. 22, 185 (1959).

2. C. K. Chen, A. R. B. de Castro, and Y. R. Chen, "Surface-enhanced second harmonic generation," Phys. Rev. Lett. 46, 145 (1981).

3. G. T. Boyd, Z. H. Yu, and Y. R. Shen, "Photoinduced luminescence from the noble metals and its enhancement on roughened surface," Phys. Rev. B 33, 7923 (1986).

4. E. Dulkeith, T. Niedereichholz, T. A. Klar, J. Feldmann, G. von Plessen, D. I. Gittins, K. S. Mayya, and F. Caruso, "Plasmon emission in photoexcited gold nanoparticles," Phy. Rev. B 70, 205424 (2004).

5. J. Wang, E. Gürdal, A. Horneber, S. Dickreuter, S. Kostcheev, A. J. Meixner, M. Fleischer, P.-M. Adam, and D. Zhang, "Carrier recombination and plasmonic emission channels in metallic photoluminescence," Nanoscale 10, 8240-8245 (2018).

6. M. B. Mohamed, V. Volkov, S. Link, and M. A. El-Sayed, "The 'lightning' gold nanorods: fluorescence enhancement of over a million compared to the gold metal," Chem. Phys. Lett. 317, 517-523 (2000).

7. R. A. Farrer, F. L. Butterfield, V. Chen, and J. T. Fourkas, "Highly efficient multiphoton-absorption-induced luminescence from gold nanoparticles," Nano Lett. 5, 1139-1142 (2005).

8. P. Mühlschlegel, H.-J. Eisler, O. J. F. Martin, B. Hecht, and D. W. Pohl, "Resonant optical antennas," Science 308, 1607 (2005).

9. M. R. Beversluis, A. Bouhelier, and L. Novotny, "Continuum generation from single gold nanostructures through near-field mediated intraband transitions," Phys. Rev. B 68, 115433 (2003).

10. J. Beermann and S. I. Bozhevolnyi, "Two-photon luminescence microscopy of field enhancement at gold nanoparticles," Phys. Status Solidi C 2, 3983-3987 (2005).

11. A. Bouhelier, R. Bachelot, G. Lerondel, S. Kostcheev, P. Royer, and G. P. Wiederrecht, "Surface plasmon characteristics of tunable photoluminescence in single gold nanorods," Phys. Rev. Lett. 95, 267405 (2005).

12. V. Knittel, M. P. Fischer, T. de Roo, S. Mecking, A. Leitenstorfer, and D. Brida, "Nonlinear photoluminescence spectrum of single gold nanostructures," ACS Nano 9, 894 (2015).

13. K. Imura, T. Nagahara, and H. Okamoto, "Imaging of surface plasmon and ultrafast dynamics in gold nanorods by near-field microscopy," J. Phys. Chem. B 108, 16344-16347 (2004).

14. P. J. Schuck, D. P. Fromm, A. Sundaramurthy, G. S. Kino, and W. E. Moerner, "Improving the mismatch between light and nanoscale objects with gold bowtie nanoantennas," Phys. Rev. Lett. 94, 017402 (2005).

15. P. Ghenuche, S. Cherukulappurath, T. H. Taminiau, N. F. van Hulst, and R. Quidant, "Spectroscopic mode mapping of resonant plasmon nanoantennas," Phys. Rev. Lett. 101, 116805 (2008). 
16. S. Viarbitskaya, A. Teulle, R. Marty, J. Sharma, C. Girard, A. Arbouet, and E. Dujardin, "Tailoring and imaging the plasmonic local density of states in crystalline nanoprisms," Nature Mater. 12, 426-432 (2013).

17. P. Biagioni, M. Celebrano, M. Savoini, G. Grancini, D. Brida, S. MátéfiTempfli, M. Mátéfi-Tempfli, L. Duò, B. Hecht, G. Cerullo, and M. Finazzi, "Dependence of the two-photon photoluminescence yield of gold nanostructures on the laser pulse duration," Phys. Rev. B 80, 045411 (2009).

18. P. Biagioni, D. Brida, J.-S. Huang, J. Kern, L. Duó, B. Hecht, M. Finazzi, and G. Cerullo, "Dynamics of four-photon photoluminescence in gold nanoantennas," Nano Lett. 12, 2941-2947 (2012).

19. X.-F. Jiang, Y. Pan, C. Jiang, T. Z. P. Yuan, T. Venkatesan, and Q.-H. Xu, "Excitation nature of two-photon photoluminescence of gold nanorods and coupled gold nanoparticles studied by twopulse emission modulation spectroscopy," J. Phys. Chem. Lett. 4, 1634-1638 (2013).

20. O. Demichel, M. Petit, S. Viarbitskaya, R. Méjard, F. de Fornel, E. Hertz, F. Billard, A. Bouhelier, and B. Cluzel, "Dynamics, efficiency, and energy distribution of nonlinear plasmon-assisted generation of hot carriers," ACS Photon. 3, 791-795 (2016).

21. R. Méjard, A. Verdy, M. Petit, A. Bouhelier, B. Cluzel, and O. Demichel, "Energy-resolved hot-carrier relaxation dynamics in monocrystalline plasmonic nanoantennas," ACS Photon. 3, 1482-1488 (2016).

22. U. Kumar, S. Viarbitskaya, A. Cuche, C. Girard, S. Bolisetty, R. Mezzenga, G. Colas des Francs, A. Bouhelier, and E. Dujardin, "Designing plasmonic eigenstates for optical signal transmission in planar channel devices," ACS Photon. 5, 2328-2335 (2018).

23. H. Wang, T. B. Huff, D. A. Zweifel, W. He, P. S. Low, and A. W. J.-X. Cheng, "In vitro and in vivo two-photon luminescence imaging of single gold nanorods," Proc. Natl. Acad. Sci. USA 102, 15752-15756 (2005).

24. J. Chen, A. Krasavin, P. Ginzburg, A. V. Zayats, T. Pullerits, and K. J. Karki, "Evidence of high-order nonlinearities in supercontinuum white-light generation from a gold nanofilm," ACS Photon. 5, 1927-1932 (2018).

25. T. Haug, P. Klemm, S. Bange, and J. M. Lupton, "Hot-electron intraband luminescence from single hot spots in noble-metal nanoparticle films," Phys. Rev. Lett. 115, 067403 (2015).

26. L. Roloff, P. Klemm, I. Gronwald, R. Huber, J. M. Lupton, and S. Bange, "Light emission from gold nanoparticles under ultrafast nearinfrared excitation: thermal radiation, inelastic light scattering, or multiphoton luminescence?" Nano Lett. 17, 7914-7919 (2017).

27. M.-B. Lien, J.-Y. Kim, M.-G. Han, Y.-C. Chang, Y.-C. Chang, H. J. Ferguson, Y. Zhu, A. A. Herzing, J. C. Schotland, N. A. Kotov, and T. B. Norris, "Optical asymmetry and nonlinear light scattering from colloidal gold nanorods," ACS Nano 11, 5925-5932 (2017).

28. J. R. Kirtley, T. N. Theis, J. C. Tsang, and D. J. DiMaria, "Hot-electron picture of light emission from tunnel junctions," Phys. Rev. B 27, 4601-4611 (1983).

29. R. Fedorovich, A. Naumovets, and P. Tomchuk, "Electron and light emission from island metal films and generation of hot electrons in nanoparticles," Phys. Rep. 328, 73-79 (2000).

30. M. Buret, A. V. Uskov, J. Dellinger, N. Cazier, M.-M. Mennemanteuil, J. Berthelot, I. V. Smetanin, I. E. Protsenko, G. Colas-des-Francs, and A. Bouhelier, "Spontaneous hot-electron light emission from electron-fed optical antennas," Nano Lett. 15, 5811-5818 (2015).
31. T. Malinowski, H. R. Klein, M. lazykov, and P. Dumas, "Infrared light emission from nano hot electron gas created in atomic point contacts," Europhys. Lett. 114, 57002 (2016).

32. D. B. Scarl, "Measurements of photon correlations in partially coherent light," Phys. Rev. 175, 1661-1668 (1968).

33. P. K. Tan, G. H. Yeo, H. S. Poh, A. H. Chan, and C. Kurtsiefer, "Measuring temporal photon bunching in blackbody radiation," Astrophys. J. Lett. 789, L10 (2014).

34. C. Kurtsiefer, P. Zarda, S. Mayer, and H. Weinfurter, "The breakdown flash of silicon avalanche photodiodes-Back door for eavesdropper attacks?" J. Mod. Opt. 48, 2039-2047 (2001).

35. E. Gamaly, Femtosecond Laser-Matter Interactions (Pan Stanford, 2011).

36. J.-S. Huang, V. Callegari, P. Geisler, C. Brüning, J. Kern, J. C. Prangsma, X. Wu, T. Feichtner, J. Ziegler, P. Weinmann, M. Kamp, A. Forchel, P. Biagioni, U. Sennhauser, and B. Hecht, "Atomically flat single-crystalline gold nanostructures for plasmonic nanocircuitry," Nat. Commun. 1, 1-8 (2010).

37. S. Grossmann, D. Friedrich, M. Karolak, R. Kullock, E. Krauss, M. Emmerling, G. Sangiovanni, and B. Hecht, "Nonclassical optical properties of mesoscopic gold," Phys. Rev. Lett. 122, 246802 (2019).

38. S. Boroviks, C. Wolff, J. Linnet, Y. Yang, F. Todisco, A. S. Roberts, S. I. Bozhevolnyi, B. Hecht, and N. A. Mortensen, "Interference in edgescattering from monocrystalline gold flakes," Opt. Mater. Express 8, 3688-3697 (2018).

39. L. Mandel and E. Wolf, Optical Coherence and Quantum Optics (Cambridge University, 1995).

40. R. Verberk and M. Orrit, "Photon statistics in the fluorescence of single molecules and nanocrystals: correlation functions versus distributions of on- and off-times," J. Chem. Phys. 119, 2214-2222 (2003).

41. F. T. Arecchi, E. Gatti, and A. Sona, "Time distribution of photons from coherent and Gaussian sources," Phys. Lett. 20, 27-29 (1966).

42. F. Boitier, A. Godard, E. Rosencher, and C. Fabre, "Measuring photon bunching at ultrashort timescale by two-photon absorption in semiconductors," Nat. Phys. 5, 267-270 (2009).

43. A. Delteil, C. Tat Ngai, T. Fink, and A. Omamoğu, "Second-order photon correlation measurement with picosecond resolution using frequency upconversion," Opt. Lett. 44, 3877-3880 (2019).

44. S. M. Novikov, C. Frydendahl, J. Beermann, V. A. Zenin, N. Stenger, V. Coello, N. A. Mortensen, and S. I. Bozhevolnyi, "White light generation and anisotropic damage in gold films near percolation threshold," ACS Photon. 4, 1207-1215 (2017).

45. Y. Qu and S. Singh, "Measurements of photon statistics in second harmonic generation," Phys. Rev. A 51, 2530-2536 (1995).

46. J. Mika, L. Podhora, L. Lachman, P. Obšil, J. Hloušek, M. Ježek, R. Filip, and L. Slodička, "Generation of ideal thermal light in warm atomic vapor," New J. Phys. 20, 093002 (2018).

47. B. Romeira and A. Fiore, "Purcell effect in the stimulated and spontaneous emission rates of nanoscale semiconductor lasers," IEEE J. Quantum Electron. 54, 1-12 (2018).

48. P. Ryczkowski, M. Barbier, A. T. Friberg, J. M. Dudley, and G. Genty, "Ghost imaging in the time domain," Nat. Photon. 10, 167-170 (2016).

49. R. H. Brown and R. Q. Twiss, "The question of correlation between photons in coherent beams of light," Nature 178, 1447-1448 (1956). 